\title{
Uso de Redes Complexas para Classificação de Doenças Pulmonares Intersticiais em Imagens de Tomografia Computadorizada
}

\author{
Yana K. D. Mendes ${ }^{1}$, Eliana S. Almeida ${ }^{1,2}$, Raquel da S. Cabral ${ }^{1,4}$, \\ Fabiane da S. Queiroz ${ }^{1,3}$ \\ ${ }^{1}$ Laboratório de Computação Científica e Análise Numérica - \\ Universidade Federal de Alagoas (UFAL) \\ CEP 57072-900 - Maceió - AL. \\ ${ }^{2}$ Faculdade de Medicina - Universidade Federal de Alagoas (UFAL) \\ CEP 57072-900 - Maceió - AL. \\ ${ }^{3}$ Centro de Ciências Agrárias - Universidade Federal de Alagoas (UFAL) \\ CEP 57100-000 - Rio Largo - AL. \\ ${ }^{4}$ Núcleo de Ciências Exatas - Universidade Federal de Alagoas (UFAL) \\ CEP 57309-005 - Arapiraca - AL. \\ ykdmeic.ufal.br, eliana.almeida@laccan.ufal.br, \\ raquel.cabraldarapiraca.ufal.br, fabiane.queiroz@ceca.ufal.br
}

\begin{abstract}
Interstitial Lung Diseases (ILDs) are characterized by the progressive healing of the pulmonary interstitium and can lead to respiratory failure. This article proposes a method of classifying DPIs based on Computed Tomography (CT) images. Each image was mapped in a complex network structure, and centrality metrics were used to obtain textural attributes of these images. Using a textit K-Nearest Neighbors (KNN) classifier, the results presented an average accuracy of $89.81 \%$. For the textural pattern of DPI of the pulmonary consolidation and ground-glass opacity type, the accuracy of the method was $90 \%$ and $86 \%$, respectively, which points to the method proposed as promising for future studies on CT images associated with patients with COVID-19.
\end{abstract}

Resumo. Doenças Pulmonares Intersticiais (DPIs) são caracterizadas pela cicatrização progressiva do interstício pulmonar e podem levar à insuficiência respiratória. Este artigo propõe um método de classificação de DPIs a partir de imagens de Tomografia Computadorizada (TC) mapeadas em uma Rede Complexa. Métricas de centralidade foram usadas com o objetivo de obter seus atributos texturais. Utilizando um classificador KNN, os resultados apresentaram uma acurácia média de $89.81 \%$. Para os padrões de textura de DPI do tipo consolidação pulmonar e opacidade em vidro fosco, a acurácia do método foi de $90 \%$ e $86 \%$, respectivamente, o que aponta o método proposto como promissor para estudos futuros em imagens de TC associadas a pacientes com COVID-19.

\section{Introdução}

As Doenças Pulmonares Intersticiais (DPIs), também denominadas doenças parenquimatosas difusas, formam um grupo com mais de 150 patologias diferentes que afetam 
a região intersticial, que inclui as paredes dos sacos aéreos dos pulmões (alvéolos) e as áreas ao redor dos vasos sanguíneos e vias aéreas inferiores [Pereyra et al. 2014]. O diagnóstico dessas patologias é estabelecido com base no histórico completo do paciente (sintomas, histórico familiar, registro da doença, etc), exame físico, exames laboratoriais e testes da função pulmonar, bem como nos achados visuais nas radiografias de tórax.

A Tomografia Computadorizada (TC) é um exame que utiliza radiação eletromagnética na faixa de raios-X para gerar imagens internas do corpo humano, que podem ser de ossos, órgãos ou tecidos. Na TC, uma série de radiografias são analisadas e imagens referentes a cortes em diferentes planos são fornecidas. A partir desses cortes, os especialistas buscam por padrões radiográficos que caracterizam as Doenças Pulmonares Intersticiais (DPIs). O conjunto de Figuras 1(a) a 1(f) ilustra os seis padrões principais de DPIs, respectivamente: normal, consolidação pulmonar, área enfisematosa, espessamento septal, favo de mel e opacidade em vidro fosco, sendo consolidação pulmonar e opacidade em vidro fosco anormalidades características de doenças como o COVID-19 [Ng et al. 2020].

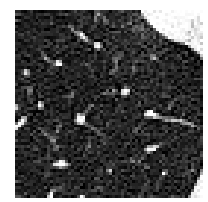

(a)

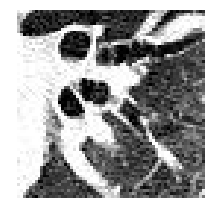

(b)

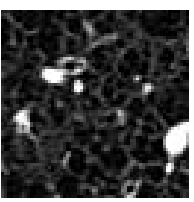

(c)

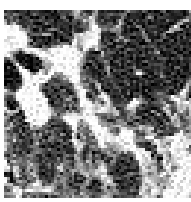

(d)

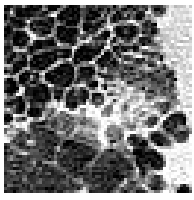

(e)

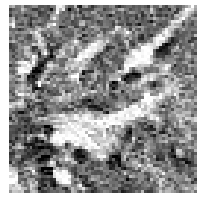

(f)

Figura 1. Seis padrões principais de DPIs. (a) Normal, (b) Consolidação pulmonar, (c) Área enfisematosa, (d) Espessamento septal, (e) favo de mel, e (f) opacidade em vidro fosco.

Recursos computacionais que envolvem técnicas de Visão Computacional têm sido utilizados para auxiliar no diagnóstico das DPIs [Pereyra et al. 2014]. Essas técnicas têm como objetivo construir sistemas para interpretar imagens, através da transformação de uma imagem em uma estrutura de dados simplificada [Gonçalves 2010]. Uma das limitações nessa área é a ausência de um algoritmo genérico para simular o processo de visão, dada as características particulares de cada problema [Gonçalves 2010].

As Redes Complexas têm recebido um crescente interesse nas mais diversas áreas do conhecimento. Esse crescimento relaciona-se diretamente à sua flexibilidade em modelar e simular estruturas topológicas que aparecem em nosso cotidiano, como por exemplo, controle de fluxos de tráfego e prevenção de colisões [Wang and Tsai 2013] e pesquisa de marketing [Yang et al. 2010]. Neste artigo apresenta-se um método automático para classificar imagens de TC que apresentam padrões de DPIs. Nesse caso, as imagens são mapeadas em um grafo e uma métrica de centralidade Closeness é aplicada adicionalmente aos valores extraídos pelos descritores Haralick [Haralick et al. 1973] e LBP(Padrão Binário Local) [Wang and He 1990], para gerar um vetor de características de textura propícias para a classificação destes padrões.

$\mathrm{O}$ artigo está estruturado da seguinte forma: $\mathrm{Na}$ Seção 2, apresenta-se trabalhados relacionados. O método proposto para classificação de textura em imagens tomográficas com DPIs é apresentado na Seção 3. Na Seção 4, são exibidos os experimentos e resultados obtidos com o método proposto, bem como a comparação com outros métodos da 
literatura. As conclusões e os trabalhos futuros são apresentados na Seção 5.

\section{Trabalhos Relacionados}

A maioria das pesquisas sobre classificação de textura em imagens visa encontrar um descritor de textura eficiente, poderoso e discriminativo [Simon and Uma 2018]. Os descritores de textura extraem atributos das imagens e os representam eficientemente, proporcionando maior precisão de classificação, independentemente do classificador utilizado [Simon and Uma 2018].

Existem diversos tipos de descritores de texturas convencionais, tais como: Padrão Binário Local (LBP - Local Binary Patterns) [Wang and He 1990], Matriz de Co-Ocorrência dos Nível de Cinza (GLCM - Gray-Level Co-Occurrence Matrix) [Haralick et al. 1973], Filtros de Gabor [Fogel and Sagi 1989], etc. Além dos descritores de textura convencionais, as redes neurais convolucionais (Convolutional Neural Network - CNN) e as Redes Complexas vêm sendo utilizadas em diferentes problemas de Visão Computacional e Processamento de Imagens. Por exemplo, [Vogado et al. 2019] usa um CNN para extrair características de textura, contudo, esse tipo de metodologia não é o foco deste trabalho.

O uso de Redes Complexas é empregado em problemas de segmentação [Xu and Uberbacher 1997], análise e classificação de texturas [de Lima et al. 2019], dentre outros. Em geral, os métodos de extração de atributos descritivos de imagens baseado em Redes Complexas utilizam a seguinte metodologia: o mapeamento da imagem em uma rede para, posteriormente, utilizar aspectos topológicos da rede na caracterização e classificação da textura. Trabalhos recentes que utilizaram essa metodologia são os de [Gonçalves et al. 2016] e [Couto et al. 2017].

No método proposto por [Gonçalves et al. 2016], uma imagem é mapeada para uma rede direcionada regular, em que cada pixel se torna um vértice da rede. Dois vértices são conectados se estão dentro de uma vizinhança determinada por um valor de raio $r$. Em seguida, uma operação de limiarização é aplicada para transformar a rede direcionada regular em uma rede direcionada escalonada, e um conjunto de arestas são removidas neste processo, a depender do valor de limiar. Vários valores de limiar são utilizados, obtendo-se assim múltiplas redes escalonadas a partir de uma única imagem. Para cada rede, caminhadas aleatórias são, por fim realizadas, afim de estimar a atividade em uma dada rede, e estes valores de atividades são representados por um histograma gerado pela associação dos valores de centralidade do grau (in-degree) dos vértices com o número de passadas do caminhante nestes mesmos vértices. Para cada rede escalonada, pela variação do limiar, os valores de histograma são concatenados, gerando assim um vetor de atributos de uma dada imagem.

Outro trabalho que aborda a caracterização de texturas com grafos foi proposto por [Couto et al. 2017]. Nesse trabalho é apresentado o descritor de textura que utiliza caminhadas determinísticas e valores in-degree dos vértices para assim gerar um vetor de características. As caminhas determinísticas são iniciadas por caminhantes que partem de um dos vértices da rede e em seguida, informações são extraídas sobre essas caminhadas. As caminhadas são chamadas de determinísticas, por que cada caminhante vai para o vértice mais próximo que não tenha sido visitado nos últimos passos.

Os trabalhos de [Gonçalves et al. 2016] e [Couto et al. 2017], mostram-se pro- 
missores e eficientes, contudo eles apresentam o problema da extração de características de textura em imagens através de aspectos locais da rede uma vez que a análise é feita considerando-se apenas valores de grau de entrada da rede. Contudo, descritores globais costumam ser mais comumente utilizados em problemas de descrição e classificação de objetos em imagens [Bharati et al. 2004]. Métodos de abordagem global descrevem a imagem como um todo para generalizar um dado objeto. A exemplo de métodos globais empregados na descrição de texturas em imagens, tem-se o tradicional LBP.

Com base nisso, buscou-se por um método mais global. Ao analisar uma rede, um aspecto importante a ser considerado consiste em descobrir quais são os vértices ou arestas que são mais importantes (ou centrais) [Lu et al. 2016]. Com a medida de centralidade closeness, pode-se obter a distância total de um vértice a todos os demais vértices da rede, e dessa forma, o closeness faz uma análise global da rede. Utilizar esse tipo de abordagem é um diferencial frente aos trabalhos de [Gonçalves et al. 2016] e [Couto et al. 2017].

\section{Método}

Nesta seção, apresenta-se o método proposto para classificação das DPIs em imagens de TC. O método está dividido em etapas, conforme ilustra a Figura 2. De modo geral, na etapa A uma imagem é mapeada em um conjunto de redes escalonadas $G_{r, t_{i}}$. Na etapa B a métrica de centralidade Closeness é aplicada com o objetivo de obter informações da textura da imagem. Para cada grafo $G_{r, t_{i}}$, um histograma com valores da métrica de centralidade é gerado. Após concatenados estes histogramas, um vetor de atributos de textura é formado com dados do histograma, e valores resultantes da aplicação dos descritores de Haralick e LBP. Por fim, na etapa C, os dados do vetor são classificados utilizando-se um classificador KNN.

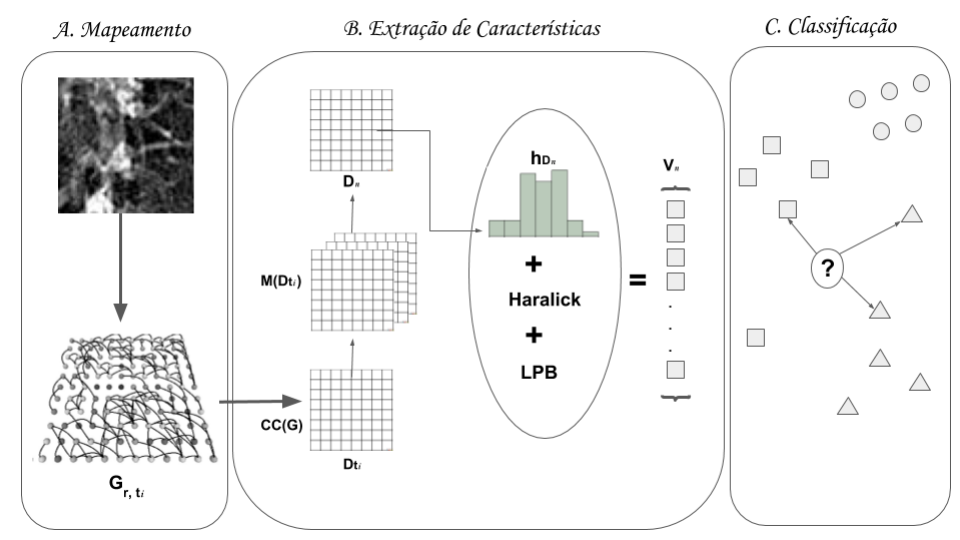

Figura 2. Principais etapas do método proposto.

\subsection{Mapeamento}

O processo de mapeamento da imagem para uma rede foi realizado com base nos métodos propostos por [Gonçalves et al. 2016] e [Couto et al. 2017]. Considerou-se que uma imagem consiste em um par $(P, I)$, onde $P$ é um conjunto finito de pixels $p_{i}$ e $I$ é o conjunto dos valores de intensidades dos pixels e está no intervalo de 0 à 255 . Durante o processo de mapeamento imagem-grafo, uma rede regular é definida inicialmente conectando-se dois vértices $v_{i}$ e $v_{j}$ se a distância euclidiana entre os pixels $p_{i}$ e $p_{j}$ for igual ou menor que um 
determinado raio $r$, pré estabelecido. O raio $r$ limita as conexões espacialmente distantes na imagem. A Figura 3 ilustra o resultado do mapeamento de um pequeno fragmento de uma imagem em rede direcionada regular. Neste trabalho, utilizou-se um valor de raio $r$ fixo, a exemplo do trabalho de [Couto et al. 2017], e não a combinação de diferentes raios como faz [Gonçalves et al. 2016].

Para cada ligação entre dois vértices $v_{i}$ e $v_{j}$, um valor de peso $e_{v_{i}, v_{j}}$ é definido pela diferença de intensidade dos pixels $p_{i}$ e $p_{j}$ e este é atribuído a aresta de acordo com a Equação 1:

$$
e_{v_{i}, v_{j}}=\left\{\begin{array}{cc}
I\left(p_{i}\right)-I\left(p_{j}\right), & \text { se } \\
N a N, & \text { caso contrário }
\end{array} \quad \operatorname{dist}\left(p_{i}, p_{j}\right) \leq r\right.
$$

em que $\operatorname{dist}\left(p_{i}, p_{j}\right)$ é a distância euclidiana entre os pixels $p_{i}$ e $p_{j}$. As direções das ligações entre os vértices $v_{i}$ e $v_{j}$ da rede são obtidas levando-se em consideração a diferença do maior para o menor valor de intensidade entre $p_{i}$ e $p_{j}$.

O próximo passo desta etapa de mapeamento consiste em aplicar um conjunto de operações para transformar a rede obtida até o momento em uma rede complexa $G$. Afim de revelar as propriedades da textura da imagem, aplicou-se uma função sobre as ligações da rede que consiste na seleção de aresta de acordo com o valor de peso $e_{v_{i}, v_{j}}$. As ligações que são mantidas na rede, são definidas pela Equação 2. Nessa equação, $t$ indica um valor de limiar que irá gerar uma versão escalonada da rede.

$$
e_{v j, v i}=\left\{\begin{array}{lc}
e_{v i, v j}, & s e \\
N a N, & \text { caso con+ }{ }^{+} \text {rio }
\end{array}\right.
$$

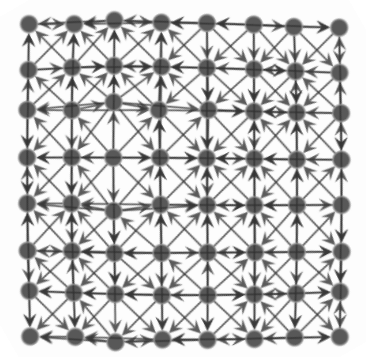

Figura 3. Exemplo de uma imagem mapeada em uma rede regular. Cada vértice está conectado com outros vértices dentro de um raio $r=2$.

Considerou-se a função do limiar como uma análise de rede em múltiplas escalas [Gonçalves et al. 2016], transformando assim uma rede regular em uma rede escalonada. Com a variação do limiar $t$, é gerado um conjunto $T=t_{1}, t_{2}, \ldots, t_{n}$, onde para cada valor de $t_{i}$ uma rede $G_{r, t_{i}} t_{i}$-escalonada é criada. Com isso, diferentes limiares são utilizados afim de analisar a evolução da rede através da adição de novas ligações. Em comparação com uma rede regular, a rede $G_{r, t_{i}}$ apresenta propriedades diferentes, pois os valores de grau de entrada e saída são diferentes em cada rede $G_{r, t_{i}}$ gerada. Além disso, revela a estrutura e a topologia relacionadas à sua escala. Para valores pequenos de $t_{i}$, a rede captura detalhes da imagem relacionados com pequenos conjuntos de pixels, à medida que $t_{i}$ aumenta, a rede expressa informações globais da imagem. Mais detalhes sobre 
o mapeamento da imagem em um conjunto de redes escalonadas, e sobre como se dá a escolha dos valores de $t_{i}$, podem ser entendidos no trabalho de [Gonçalves et al. 2016]. A Figura 4 mostra exemplos de redes depois de aplicada a função de limiarização.

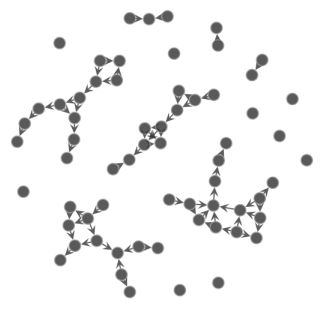

(a) $t_{1}=10$

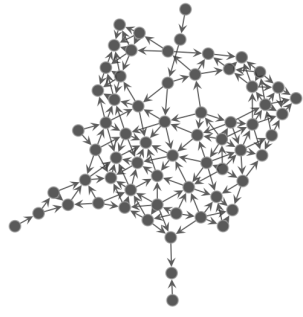

(b) $t_{2}=50$

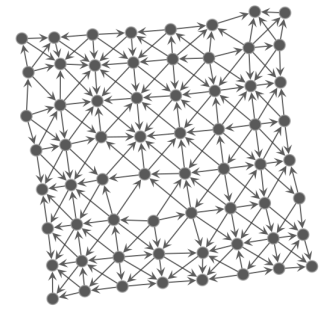

(c) $t_{3}=150$

Figura 4. Exemplo de redes $G_{r, t_{i}}$ escalonadas para diferentes valores de limiar $t_{i}$ considerando um raio $r$.

Após o mapeamento da imagem para o conjunto de redes complexas $G_{r, t_{i}}$, é necessário caracterizá-las de forma robusta e eficiente. Essa caracterização pode ser realizada por meio das diversas métricas propostas para redes complexas (média do grau de entrada dos vértices, coeficiente de aglomeração, dentre outras métricas). Entretanto, nos experimentos pode-se observar que a medida de centralidade Closeness apresentou um alto desempenho e aplicabilidade na resolução do problema aqui proposto.

A medida de centralidade Closeness é calculada com base no número de caminhos mínimos de um vértice em relação ao outros vértices do grafo [Lu et al. 2016]. Para um grafo conexo $G$, a medida Closeness de um vértice $v_{i}$ é dada pelo inverso das somas das distâncias dos caminhos mínimos de $v_{i}$ para todos os outros vértices da rede. No entanto, essa definição tem não se aplica quando a rede é desconexa, pois alguns vértices não são alcançáveis. Assim, uma forma mais comum de calcular essa medida de centralidade é usar a soma dos inversos das distâncias dos caminhos mínimos. Dessa forma, o Closeness é dado por:

$$
C C(i)=\frac{1}{N-1} \sum_{j \neq i} \frac{1}{d_{i j}},
$$

onde $N$ é o número de vértices do grafo; $d_{i j}$ é a distância entre os vértices $i$ e $j$; e $\frac{1}{d_{i j}}=0$ senão existe caminho entre $i$ e $j$.

Deste modo, quanto maior o valor de Closeness, mais central é o vértice. Em geral, o vértice com o maior valor de Closeness tem a melhor visão do fluxo de informações [Lu et al. 2016]. Diferente dos trabalhos de [Gonçalves et al. 2016] e [Couto et al. 2017], que utilizam valores de grau de entrada dos vértices da rede, os valores obtidos pela métrica Closeness pode ser visto como um descritor global de texturas, uma vez que o valor da Closeness para cada vértice é calculado considerando todos os outros vértices da rede.

Dessa forma, após a obtenção do conjunto das redes escalonadas $G_{r, t_{i}}$, aplicou-se o Closeness nessas redes, gerando uma matriz (mapas de valores do Closeness) $D_{t_{i}}$ para cada uma das redes. A Figura 5 mostra os seis padrões radiográficos (Figura 5(a)-(f)) e suas respectivas imagens geradas a partir da medida de centralidade Closeness (Figura 5(g)-(l)). Conforme pode-se perceber na Figura 5, com o valor do limiar $t=270$, a rede 
$G_{r, t_{i}}$ captura mais detalhes da textura da imagem. Além disso, mais ligações na rede são mantidas e, portanto tem-se mais vértices com alto valor de centralidade, resultando em um mapa de valores que expressam mais detalhes sobre textura. A Figura 5(e), por exemplo, ilustra o padrão radiográfico favo de mel e, este padrão é traduzido nas imagens de TC como espaços císticos agrupados [Souza et al. 2002]. E este mesmo padrão pode ser visto na Figura 5(k), que foi gerada através da medida de centralidade closeness.

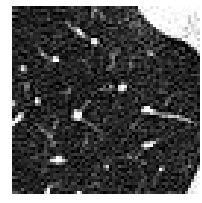

(a)

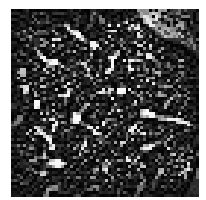

(g)

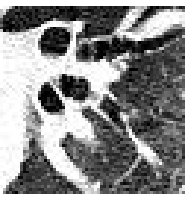

(b)

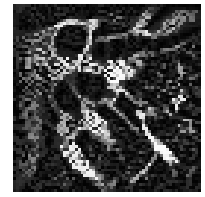

(h)

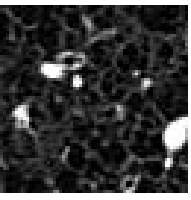

(c)

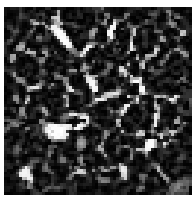

(i)

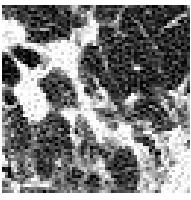

(d)

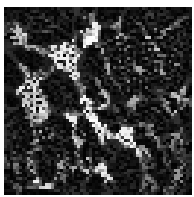

(j)

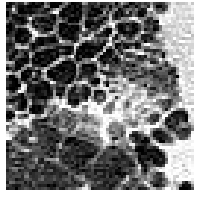

(e)

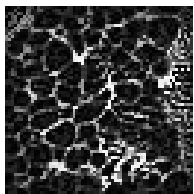

(k)

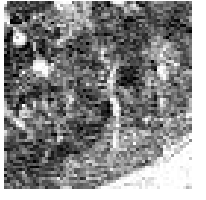

(f)

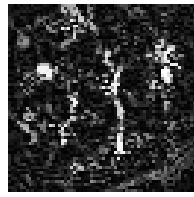

(l)

Figura 5. Exemplos da aplicação da medida de centralidade closeness nos seis padrões radiográficos. A rede foi escalonada com de $t=270$ e raio de valor fixo $r=5$.

\subsection{Definição do Vetor de Atributos de Texturas}

Conforme observado na seção anterior, os valores de centralidade dos vértices incorporam informações importantes sobre a textura. Para agrupar essa medida, é proposto um vetor de características composto pela relação dos níveis de cinza da imagem e os valores obtidos pela medida de centralidade closeness.

Antes de gerar o vetor $V$ de atributos de texturas, calculou-se a média dos valores da centralidade obtidos em todos os mapas $D_{t_{i}}$, gerando uma única matriz $D$ para cada imagem. Após a obtenção de $D$, objetivando gerar o vetor de atributos de texturas, realizou-se uma analise quanto a relação entre os níveis de cinza da imagem e os valores obtidos com closeness. Para tanto, verificou-se o nível de cinza do pixel $p_{i}$. Caso este nível esteja no intervalo $w<I\left(p_{i}\right) \leq z$ então, o valor do closeness $(C C(i))$ no vértice referente ao pixel $p_{i}$ é somado com os demais valores de closeness dos pixels com a mesma intensidade do pixel $p_{i}$ e que estavam no mesmo intervalo $\left[w_{m}, z_{m}\right]$. Esses valores são armazenados em $S_{w_{m}, z_{m}}$, onde $m$ é a quantidade de intervalos utilizados (Equação 4).

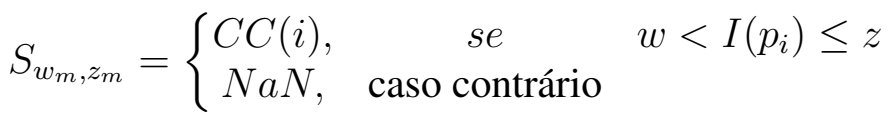

Essa análise é feita para todas as imagens e para cada uma delas tem-se um vetor com valores de closseness para todos os intervalos $\left[w_{m}, z_{m}\right]$ :

$$
\varphi^{n}=\left[S_{w_{1}, z_{1}}^{n}, S_{w_{2}, z_{2}}^{n}, S_{w_{3}, z_{3}}^{n}, \ldots, S_{w_{m}, z_{m}}^{n}\right]
$$

onde $n$ é a quantidade de imagens existentes na base de dados. 
O vetor de características com os valores da medida de centralidade foi criado concatenando os vetores $\varphi^{n}$ :

$$
\varphi=\left[\varphi^{1} ; \varphi^{2} ; \varphi^{3} ; \ldots, \varphi^{n}\right]
$$

Cada $\varphi^{n}$ representa os valores de closeness para uma dada imagem da base de dados.

Além das características extraídas pelo método proposto, percebeu-se a necessidade de extrair mais informações globais da imagem. Para tanto, escolheu-se o LBP e os descritores de Haralick, que são dois métodos clássicos da literatura e que conseguem descrever bem as texturas das imagens. Dessa forma, aplicou-se os dois métodos nas imagens de tomografia com DPIs. As características do método proposto, juntamento com o LBP e os descritores de Haralick formaram um vetor $(V=\varphi+$ Haralick $+L B P)$ que contém as informações de todas as imagens. $\mathrm{O}$ vetor de características final será utilizado em um processo de classificação.

\section{Resultados e Discussões}

A base de dados utilizada nesse trabalho foi retirada de um banco de dados contendo 247 imagens de tomografia com DPIs, selecionadas por um grupo de radiologistas, de 108 diferentes exames realizados no Hospital das Clínicas da Faculdade de Medicina de Ribeirão Preto da Universidade de São Paulo (HCFMRP - USP) [Pereyra et al. 2014].

Dessas 247 imagens, foram selecionados 3.258 regiões de interesse (Regios Of Interest - ROIs) a partir do recorte automático das imagens em regiões com tamanhos $64 \times$ 64 pixels. Esses ROIs foram agrupados em categorias de acordo com o relato radiológico e o padrão radiográfico presente. São elas: favo de mel, vidro fosco, espessamento septal, consolidação pulmonar, áreas enfisematosas e normal.

Para realizar a classificação dos dados obtidos, utilizou-se o classificador Kvizinhos mais próximos (K-Nearest Neighbor - KNN) com distância Euclidiana e a validação cruzada com 10 subconjuntos. Mais informações sobre o classificador e o esquema de validação cruzada encontram-se no trabalho de [Liu and Fieguth 2012]. Assim como [Gonçalves et al. 2016], escolheu-se um classificador simples para evidenciar a importância dos dados obtidos utilizando o método proposto na tarefa de classificação.

O método proposto foi executado utilizando os seguintes parâmetros: limiar inicial, $t_{0}=20$; o incremento sobre o valor atual do limiar $t_{i}=40$; número de vezes que o limiar é incrementado, $m=5$; e o raio que define a vizinhança de um vértice $v_{i}, r=4$. Segundo [Gonçalves 2010], os principais parâmetros da construção do grafo são o raio $r$ e o limiar inicial $t_{0}$. Isso ocorre porque o raio é o parâmetro que determina a área de conexão de cada vértice, e $t_{0}$ limita a escala da análise do método. O limiar $t_{i}$ é utilizado para fornecer as redes em múltiplas escaldas. Para [Gonçalves et al. 2016], valores altos de $t_{i}$ permitem que o método explore uma melhor gama de escalas na análise de rede em múltiplas escalas. Dessa forma, para este trabalho, o valor do limiar $t_{i}=40$ é o valor mais alto que conseguiu uma melhor exploração das redes em múltiplas escalas. Esses parâmetros foram escolhidos com base em experimentos realizados para encontrar valores que melhor descrevessem as imagens de tomografia com DPIs (ver detalhes sobre estes parâmetros e como realizar os experimentos em [Gonçalves et al. 2016]). Na classificação, foram realizadas 100 repetições para obter a média da acurácia obtida pelo 
$K N N$. Para validar os dados, utilizou-se a técnica de validação cruzada $k$-fold com 10 folds.

Os experimentos foram executados em uma máquina com processador Core i3, 4GB de memória RAM e Sistema Operacional GNU/Linux Ubuntu 18.04.1 LTS. Não foram utilizados recursos de GPU neste processo. O algoritmo foi implementado na linguagem de programação Python, usando pacote graph-tool para gerar os grafos e para calcular a métrica de centralidade closeness. O tempo de execução do algoritmo para todas as imagens tratadas foi em média de 7 horas e 30 minutos. O algoritmo do closeness do graph-tool tem complexidade computacional de $O(V(V+E))$, onde $V$ é o número de vértices e $E$ o número de arestas da rede.

Com esses parâmetros e recursos, obteve-se uma taxa de classificação de $79.79 \%$ no conjunto de dados gerado pelo método, utilizando o closeness, sem considerar a inserção dos descritores de Haralick e LBP no vetor. Com a junção dos três métodos, closeness, descritores de Haralick e LBP, obteve-se uma acurácia de 89.81\%. Para chegar a esse resultado, realizou-se a junção do vetor de característica final do método com o closeness com os métodos LBP e descritores de Haralick de forma individual, a junção dos dois métodos sem o closeness e a junção dos três métodos. A Tabela 1 exibe as combinações feitas e quais foram as acurácias obtidas.

Tabela 1. Resultado dos experimentos realizados com a junção dos métodos.

\begin{tabular}{lc}
\hline Métodos & Acurácia \\
\hline Haralick + LBP & 83.75 \\
Closeness + Haralick & 83.81 \\
Closeness + LBP & 88.35 \\
Closeness + Haralick + LBP & 89.81 \\
\hline
\end{tabular}

A Tabela 2 apresenta a quantidade de características existentes em cada vetor, a sensibilidade, a especificidade e acurácia para os métodos testados nesse trabalho. Podese observar que o método proposto supera os métodos clássicos da literatura, GLCM, LBP e Haralick, tanto no valor da taxa de classificação, quanto nos valores de sensibilidade e especificidade.

Dessa forma, verifica-se que, utilizando o classificador KNN, os métodos mais tradicionais funcionam de forma mais limitada, enquanto o método proposto consegue descrever de forma mais detalhada a textura das imagens de tomografia com DPIs. Isso acontece por que o closeness consegue descrever com detalhes os padrões radiográficos, e dessa forma, o classificador consegue distinguir bem um tipo de padrão radiográfico do outro. Além disso, os valores de sensibilidade e especificidade do método proposto, comprovam que o método consegue identificar quais imagens contém realmente as DPIs e quais não contém.

Como foi observado, experimentalmente, a criação de um histograma composto não apenas de valores resultantes da aplicação da medida de centralidade, mas também com valores resultantes da aplicação dos descritores Haralick e LBP, potencializa ainda mais o método. Com isso, alcançou-se uma taxa de classificação mais alta do que o método de [Pereyra et al. 2014] e de [Gonçalves et al. 2016], que são métodos mais atuais 
Tabela 2. Comparação do método proposto com outros métodos.

\begin{tabular}{lcccc}
\hline Métodos & Características & Sensibilidade & Especificidade & Acurácia \\
\hline GLCM & 4 & $71.40 \%$ & $94.87 \%$ & $66.28 \%$ \\
LBP & 10 & $69.62 \%$ & $97.90 \%$ & $72.87 \%$ \\
Descritores de Haralick & 14 & $80.93 \%$ & $96.47 \%$ & $74.22 \%$ \\
[Pereyra et al. 2014] & 16 & $90.40 \%$ & $97.0 \%$ & $82.62 \%$ \\
[Gonçalves et al. 2016] & 132 & $87.14 \%$ & $97.96 \%$ & $82.98 \%$ \\
Método Proposto & $\mathbf{9 9}$ & $\mathbf{9 1 . 3 5 \%}$ & $\mathbf{9 8 . 4 0 \%}$ & $\mathbf{8 9 . 8 1 \%}$ \\
\hline
\end{tabular}

para extração de textura. O método proposto neste trabalhou utilizou a mesma base de dados utilizada por [Pereyra et al. 2014], porém o método proposto por eles não obteve uma taxa de classificação mais alta. Isso aconteceu porque eles utilizaram apenas uma abordagem estatística. Esse tipo de abordagem não descreve bem a textura das imagens, pois é uma abordagem local. Dessa forma, percebe-se como o método proposto neste trabalho, que utiliza uma abordagem mista, consegue classificar melhor as imagens com DPIs.

A Tabela 3 exibe a matriz de confusão do KNN. Os padrões radiográficos, que são as classes utilizadas para a classificação, estão representados por letras: A - consolidação, B - enfisema, C - espessamento, D - Favo de mel, E - normal e F - vidro fosco. A matriz de confusão mostra o número de classificações corretas, que se encontram nas linhas, versus as classificações preditas, que se encontram nas colunas, para cada classe. Sabendo disso, percebe-se, por exemplo, que o modelo classificou 407 instâncias como classe A. Porém, erroneamente classificou 4 instancias da classe A como classe B, 18 instâncias como classe C, 6 instâncias como classe D, 7 instâncias como classe E e 9 instâncias como classe F. O número de acertos, para cada classe dos padrões radiográficos, localizase na diagonal principal da matriz. Percebe-se que todas as classes têm uma taxa de classificação correta acima de $80 \%$, sendo a classe A (consolidação) com a maior taxa de acerto e a classe $\mathrm{C}$ (espessamento) com a menor.

Tabela 3. Matriz de confusão com o resultado da classificação do KNN.

\begin{tabular}{c|cccccc|ccc}
\hline & $\mathrm{A}$ & $\mathrm{B}$ & $\mathrm{C}$ & $\mathrm{D}$ & $\mathrm{E}$ & $\mathrm{F}$ & Total & Correto & Erro \\
\hline $\mathrm{A}$ & $\mathbf{4 0 7}$ & 4 & 18 & 6 & 7 & 9 & 451 & $90 \%$ & $10 \%$ \\
$\mathrm{~B}$ & 5 & $\mathbf{4 3 6}$ & 12 & 0 & 49 & 0 & 502 & $87 \%$ & $13 \%$ \\
$\mathrm{C}$ & 39 & 31 & $\mathbf{4 2 3}$ & 39 & 34 & 23 & 590 & $81 \%$ & $19 \%$ \\
$\mathrm{D}$ & 4 & 27 & 48 & $\mathbf{4 3 6}$ & 5 & 10 & 530 & $82 \%$ & $18 \%$ \\
$\mathrm{E}$ & 16 & 44 & 19 & 2 & $\mathbf{4 9 8}$ & 11 & 590 & $84 \%$ & $16 \%$ \\
$\mathrm{~F}$ & 31 & 1 & 43 & 8 & 2 & $\mathbf{5 1 0}$ & 595 & $86 \%$ & $14 \%$ \\
Correto & $\mathbf{4 0 7}$ & $\mathbf{4 3 6}$ & $\mathbf{4 2 3}$ & $\mathbf{4 3 6}$ & $\mathbf{4 9 8}$ & $\mathbf{5 1 0}$ & $\mathbf{3 . 2 5 8}$ & $\mathbf{8 9 . 1 0} \%$ & $\mathbf{1 0 . 9 0 \%}$ \\
\hline
\end{tabular}

A partir da matriz confusão, pode-se perceber uma taxa de acerto de $90 \%$ e $86 \%$ para os padrões consolidação pulmonar e opacidade em vidro fosco, respectivamente. E valores de $90 \%$ e $85 \%$ de sensibilidade, e $96 \%$ e $97 \%$ de especificidade para ambos os padrões. Estes padrões radiográficos, são anomalias que podem estar presente em 
imagens de TC de pacientes com COVID-19 [Ng et al. 2020]. Diante disso, sugere-se que o método proposto também pode ter uma alta taxa de classificação, sensibilidade e especificidade para as imagens de TC advindas dos pacientes com COVID-19.

Ademais, em seu trabalho, [Pereyra et al. 2014] descrevem a DPI como uma doença que tem uma classificação consistentemente ruim, ou seja, a classificação das DPIs não é trivial, pois as características de alguns dos padrões radiográficos se confundem. Entretanto, o método proposto nesse trabalho melhora a taxa de classificação em mais de $6 \%$ em relação aos resultados anteriores. E com isso, percebe-se que as transformações realizadas nas redes complexas e a medida de centralidade closeness auxiliam na caracterização das texturas nas imagens de tomografia com DPIs.

\section{Conclusão}

Apresentou-se neste trabalho uma abordagem que extrai características da textura das imagens de tomografia com DPIs, mapeando as imagens em redes direcionadas. Para isso, considerou-se cada pixel como um vértice da rede e conectando vértices dentro de um determinado raio. Depois da rede formada e escalonada, aplicou-se a medida de centralidade closeness e formou-se um vetor de características com o somatório das centralidades que estavam dentro de um determinado intervalo.

A partir das análises dos resultados, conclui-se que o método proposto consegue descrever a textura das imagens. De forma individual, conseguiu-se uma acurácia acima dos métodos tradicionais, e com a junção dos métodos LBP e descritores Haralick foi possível subir ainda mais a taxa de classificação, com uma acurácia acima dos métodos atuais. O aumento da acurácia com a junção dos métodos se deu por causa da boa representatividade que o LBP e descritores Haralick tem em extrair informações, de forma global, nas imagens. Dessa forma, afirma-se que o método proposto, tem grande representatividade na extração de características de textura de imagens, mais especificamente nas imagens com DPIs, que são doenças que têm uma classificação consistentemente ruim.

Os bons resultados obtidos pelo método sugerem que novos trabalhos podem ser gerados. O método proposto pode ser estendido e ser aplicado em imagens coloridas ou em outros tipos de imagens médicas, como imagens de endoscopia, colonoscopia, angiografia. Além disso, o método pode ainda ser aplicado em outros problemas de Visão Computacional, como por exemplo, restauração de imagens com borramento, reconhecimento de textura em vídeos, dentre outros. E mais uma aplicação recente para o método, são em imagens de TC de pacientes com COVID-19, visto que a TC é uma modalidade de imagem mais sensível para investigação para esta doença [ $\mathrm{Ng}$ et al. 2020].

\section{Referências}

Bharati, M. H., Liu, J. J., and MacGregor, J. F. (2004). Image texture analysis: Methods and comparisons. Chemometrics and Intelligent Laboratory Systems, 72(1):57-71.

Couto, L. N., Backes, A. R., and Barcelos, C. A. Z. (2017). Texture characterization via deterministic walks' direction histogram applied to a complex network-based image transformation. Pattern Recognition Letters.

de Lima, G. V., Saito, P. T., Lopes, F. M., and Bugatti, P. H. (2019). Classification of texture based on Bag-of-Visual-Words through complex networks. Expert Systems with Applications, 133:215-224. 
Fogel, I. and Sagi, D. (1989). Gabor filters as texture discriminator. Biological Cybernetics, 61(2):103-113.

Gonçalves, W. N. (2010). Caminhadas Determinísticas em Redes Complexas Aplicadas em Visão Computacional. PhD thesis, Universidade de São Paulo - USP.

Gonçalves, W. N., Silva, N. R., Costa, L. F., and Bruno, O. M. (2016). Texture recognition based on diffusion in networks. Information Sciences, 365:51-71.

Haralick, R., Shanmugan, K., and Dinstein, I. (1973). Textural features for image classification. IEEE Transactions on Systems, Man and Cybernetics, 3.

Liu, L. and Fieguth, P. (2012). Texture Classification from Random Features. IEEE Transactions on Pattern Analysis and Machine Intelligence, 34(3):574-586.

Lu, L., Chen, D., Ren, X. L., Zhang, Q. M., Zhang, Y. C., and Zhou, T. (2016). Vital nodes identification in complex networks. Physics Reports, 650:1-63.

Ng, M.-Y., Lee, E., Yang, J., Yang, F., Li, X., Wang, H., Lui, M., Lo, C. S., Leung, B., Khong, P.-L., Hui, C., Yuen, K.-Y., and Kuo, M. D. (2020). Imaging Profile of the COVID-19 Infection: Radiologic Findings and Literature Review. Radiology: Cardiothorac Imaging., pages 1-26.

Pereyra, L. C., Rangayyan, R. M., Ponciano-Silva, M., and Azevedo-Marques, P. M. (2014). Fractal analysis for computer-aided diagnosis of diffuse pulmonary diseases in HRCT images. IEEE MeMeA 2014 - IEEE International Symposium on Medical Measurements and Applications, Proceedings.

Simon, P. and Uma, V. (2018). Review of Texture Descriptors for Texture Classification. Data Engineering and Intelligent Computing, Advances in Intelligent Systems and Computing 542,, 542:159-176.

Souza, A. S., de Araujo Neto, C., Jasinovodolinsky, D., Marchiori, E., Kavakama, J., Irion, K. L., Nobre, L. F., Funari, M., Chaves, M., Terra Filho, M., and Daltro, P. (2002). Terminologia para a descrição de tomografia computadorizada do tórax: Sugestões iniciais para um consenso brasileiro. Radiologia Brasileira, 35(2):125-128.

Vogado, L. H. S., Veras, R. M. S., Araujo, F. H. D., Silva, R. R. V., and Aires, K. R. (2019). Rede Neural Convolucional para o Diagnóstico de Leucemia. XIX Simpósio Brasileiro de Computação Aplicada à Saúde, pages 46-57.

Wang, C. and Tsai, H. (2013). Detecting urban traffic congestion with single vehicle. In 2013 International Conference on Connected Vehicles and Expo (ICCVE), pages 233-240.

Wang, L. and He, D.-C. (1990). Texture classification using texture spectrum. Pattern Recognition, 23(8):905-910.

$\mathrm{Xu}$, Y. and Uberbacher, E. C. (1997). 2D image segmentation using minimum spanning trees. ELSEVIER - Image and Vision Computing, 15:47-57.

Yang, J., Yao, C., Ma, W., and Chen, G. (2010). A study of the spreading scheme for viral marketing based on a complex network model. Physica A: Statistical Mechanics and its Applications, 389(4):859-870. 